\title{
Thyroid Hormones, Autoantibodies, Ultrasonography, and Clinical Parameters for Predicting Thyroid Cancer
}

\author{
Lin-zheng He, ${ }^{1,2}$ Tian-shu Zeng, ${ }^{1}$ Lin Pu, ${ }^{2}$ Shi-xiu Pan, ${ }^{1}$ Wen-fang Xia, ${ }^{1}$ and Lu-lu Chen ${ }^{1}$ \\ ${ }^{1}$ Department of Endocrinology, Union Hospital, Tongji Medical College, Huazhong University of Science and Technology, \\ Wuhan 430022, China \\ ${ }^{2}$ Department of Endocrinology, Chengdu First People's Hospital, Chengdu 610041, China
}

Correspondence should be addressed to Tian-shu Zeng; tszeng@hust.edu.cn

Received 19 January 2016; Accepted 23 March 2016

Academic Editor: Diego Russo

Copyright (C) 2016 Lin-zheng He et al. This is an open access article distributed under the Creative Commons Attribution License, which permits unrestricted use, distribution, and reproduction in any medium, provided the original work is properly cited.

\begin{abstract}
Our objective was to evaluate thyroid nodule malignancy prediction using thyroid function tests, autoantibodies, ultrasonographic imaging, and clinical data. We conducted a retrospective cohort study in 1400 patients with nodular thyroid disease (NTD). The thyroid stimulating hormone (TSH) concentration was significantly higher in patients with differentiated thyroid cancer (DTC) versus benign thyroid nodular disease (BTND) $(p=0.004)$. The receiver operating characteristic curve of TSH showed an AUC of 0.58 (95\% CI $0.53-0.62, p=0.001)$, sensitivity of $74 \%$, and specificity of $57 \%$ at a cut-off of $1.59 \mathrm{mIU} / \mathrm{L}$. There was an incremental increase in TSH concentration along with the increasing tumor size $(p<0.001)$. Thyroglobulin antibody (TgAb) concentration was associated with an increased risk of malignancy $(p=0.029)$, but this association was lost when the effect of TSH was taken into account $(p=0.11)$. Thyroid ultrasonographic characteristics, including fewer than three nodules, hypoechoic appearance, solid component, poorly defined margin, intranodular or peripheral-intranodular flow, and punctate calcification, can be used to predict the risk of thyroid cancer. In conclusion, our study suggests that preoperative serum TSH concentration, age, and ultrasonographic features can be used to predict the risk of malignancy in patients with NTD.
\end{abstract}

\section{Introduction}

The appearance of a thyroid nodule is a frequent occurrence. In the general population, thyroid nodules are found in $4 \%$ to $7 \%$ of adults through palpation and in $19 \%$ to $67 \%$ through ultrasonography (US). They are most frequently observed in women and in the elderly, and their prevalence is expected to continue to increase $[1,2]$. A large-scale thyroid disease epidemiological investigation in China, the most populous country in the world, has shown that the incidence of thyroid nodules increased from $10.2 \%$ in 2006 to $18.6 \%$ in 2010 .

Although thyroid cancer accounts for only about $1 \%$ of all neoplasms, it is the leading cancer site in the endocrine system, and the incidence rate is increasing faster than that of any other malignancy in both men and women, especially differentiated thyroid microcarcinomas (DTMCs), which are tumors $\leq 1 \mathrm{~cm}$ in size. Although DTMCs exhibit a more benign behavior relative to thyroid cancers of larger size
(TCLS), there is a subgroup of DTMCs that can be aggressive, requiring therapeutic management similar to TCLS [3].

As a well-established growth factor for thyroid cells, TSH can stimulate the growth of not only normal but also malignant thyroid tissues [4-6]. Current clinical management guidelines emphasize the important role for TSH suppression in the management of patients with high risk thyroid tumors $[7,8]$. Recently a number of studies have attempted to address the question of whether TSH exerts an influence on the development of thyroid cancer. A number of studies have shown that serum TSH concentration is an independent risk predictor for the development of thyroid cancer, the progression of thyroid cancer, or both [9-13]. However, there are still some opposing results $[14,15]$. Therefore, additional evidence is needed to clarify this question.

In this study, we retrospectively reviewed the records of all patients with one or more thyroid nodules. Our goal was to 
evaluate thyroid nodule malignancy prediction using thyroid function tests, autoantibodies, US imaging, and clinical data.

\section{Subjects and Methods}

Between June 2008 and December 2010, 1650 patients underwent thyroid surgery for NTD at Wuhan Union Hospital, Tongji Medical College, Huazhong University of Science and Technology. All patients were Chinese nationals, and most of them came from Hubei province in China, where median children urine iodine concentration was higher than $197.5 \mu \mathrm{g} / \mathrm{L}$ between 2005 and 2011. Among these, 1400 patients (men, 267; women, 1133; mean age $47.72 \pm 12.69$ years) who were confirmed to have a solitary thyroid nodule or a multiple nodules on an ultrasound scan and who were not known to have thyroid cancer or hyperthyroidism due to Graves' disease were included in the present study.

Patients' age, sex, history of taking levothyroxine or antithyroid drugs, preoperative serum TSH concentration, preoperative TPOAb and TgAb concentration, ultrasonographic features, and pathologic data were recorded.

The measurements of serum thyroid function tests were performed with an automated immunochemiluminescent assay (Elecsys 2010, Roche Diagnostics, Manheim, Germany). The normal range for $\mathrm{TSH}$, free triiodothyronine (FT3), and free thyroxine (FT4) was $0.4-4.0 \mathrm{mIU} / \mathrm{L}$ (sensitivity $0.01 \mathrm{mIU} / \mathrm{L}$ ), $12-22 \mathrm{pmol} / \mathrm{L}$ (sensitivity $0.01 \mathrm{pmol} / \mathrm{L}$ ), and $0.27-4.20 \mathrm{pmol} / \mathrm{L}$ (sensitivity $0.01 \mathrm{pmol} / \mathrm{L}$ ), respectively. The measurements of TPOAb and TgAb were also performed with an automated immunochemiluminescent assay (Elecsys 2010, Roche Diagnostics, Manheim, Germany). The normal ranges for TPOAb and $\mathrm{TgAb}$ were $0-34 \mathrm{IU} / \mathrm{mL}$ and $0-$ $115 \mathrm{IU} / \mathrm{mL}$, and the analytical sensitivities for TPOAb and $\mathrm{TgAb}$ were $5 \mathrm{IU} / \mathrm{mL}$ and $10 \mathrm{IU} / \mathrm{mL}$, respectively. A titer of greater than the upper limit was defined as positive. Thyroid US was always performed by one of three operators, each with special expertise in thyroid sonography, using HV 900 color HI VISION 900 US system machines (Hitachi Medical, Tokyo, Japan) and a 6-13 MHz linear array transducer.

The final diagnosis of thyroid nodules was dependent on the postoperative histology. Statistical analysis was performed to determinate whether there were differences in the recorded characteristics between patients diagnosed with benign lesions compared with those diagnosed with thyroid cancer.

Differences in the frequencies of single variables were tested with the chi-square test or independent-samples $t$ test. Binary logistic regression analysis was used to identify the independent factors associated with thyroid malignancy. Values were either reported as the mean \pm SD or odds ratio (OR) and 95\% confidence intervals (CI). In all instances, $p<$ 0.05 was considered significant. All data were analyzed using SPSS software for Windows (version 17.0).

\section{Results}

3.1. Patients and Tumor Characteristics. The final pathology data showed no evidence of malignancy in 1105 patients (78.9\%), whereas malignant lesions were present in 295
TABLE 1: Sex and age of thyroid cancer patients.

\begin{tabular}{lccc}
\hline & Malignancy (\%) & No malignancy $(\%)$ & $p$ value \\
\hline$N$ & $295(21.07 \%)$ & $1105(78.93 \%)$ & \\
Sex & & & 0.197 \\
$\quad$ Men & $64(23.97 \%)$ & $203(76.03 \%)$ & \\
$\quad$ Women & $231(20.39 \%)$ & $902(79.61 \%)$ & \\
Mean age & $44.33 \pm 13.54$ years & $48.71 \pm 12.34$ years & $<0.001$ \\
\hline
\end{tabular}

patients (21.1\%), including 178 papillary thyroid carcinomas, 104 papillary thyroid microcarcinomas, 2 follicular thyroid carcinomas, 4 lymphomas, 2 anaplastic carcinomas, 4 medullary carcinomas, and 4 metastatic carcinomas.

A disproportionate number of women relative to men (1133 : 267) underwent thyroid surgery. Men were more likely to suffer from thyroid cancer than women; 64 of the 267 male patients $(23.97 \%)$ had malignancy on final pathology versus 231 of the 1133 female patients (20.39\%), but it was not statistically significant ( $p=0.197)$. Patients with malignancy were significantly younger than those without malignancy; the mean age at the time of surgery of the patients with malignancy was $44.33 \pm 13.54$ years and the mean age of the patients without malignancy was $48.71 \pm 12.34$ years $(p<$ 0.001) (Table 1).

Significant increases in the prevalence of malignancy were detected in patients who were younger than 40 years of age ( $p<0.001$, compared with the 40-49-year group) and in those older than 70 years $(p=0.036$, compared with the 60-69-year group) (Figure 1).

3.2. TSH with the Prevalence of DTC. Patients without an available serum TSH concentration within a week before surgery (294 patients), with a final malignancy other than DTC (medullary thyroid cancer, anaplastic thyroid cancer, lymphoma, and metastatic carcinoma, 11 patients), or with a history of taking levothyroxine or antithyroid drugs (129 patients) were not included in the next statistical analysis for TSH. Finally, the remaining 985 patients were eligible for inclusion in the study.

To decrease the likelihood of patients with markedly elevated TSH skewing the data, we excluded all patients with TSH out of the normal range when comparing the mean TSH concentration between different groups. Among the remaining 794 patients, the preoperative mean TSH concentration was significantly higher in patients with DTC versus BTND $(2.10 \pm 0.07 \mathrm{mIU} / \mathrm{L}$ versus $1.86 \pm 0.04 \mathrm{mIU} / \mathrm{L}$, $p=0.004)$. If the patients treated with levothyroxine were not excluded, the difference was still statistically significant $(p=0.006)$. In addition, levothyroxine-treated patients with respect to untreated patients showed a marked, but not statistically significant, reduction in the prevalence of DTC ( $16.98 \%$ versus $20.48 \%, p=0.536$ ).

The DTC group was subdivided into DTMCs and TCLS based on the final histological diagnosis. Comparing among the three groups (DTMCs, TCLS, and BTND), the result showed that the patients with BTND had the lowest TSH concentrations, those with DTMCs had intermediate concentrations, and those with TCLS had the highest TSH 


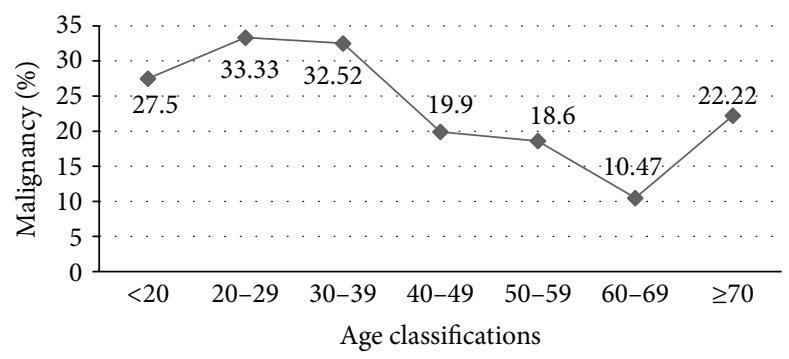

FIGURE 1: Prevalence of malignancy relative to patient age. Significant increases in the prevalence of malignancy were detected in patients who were younger than 40 years of age ( $p<0.001$, compared with the $40-49$-year group) and in those older than 70 years $(p=0.036$, compared with the 60-69-year group).

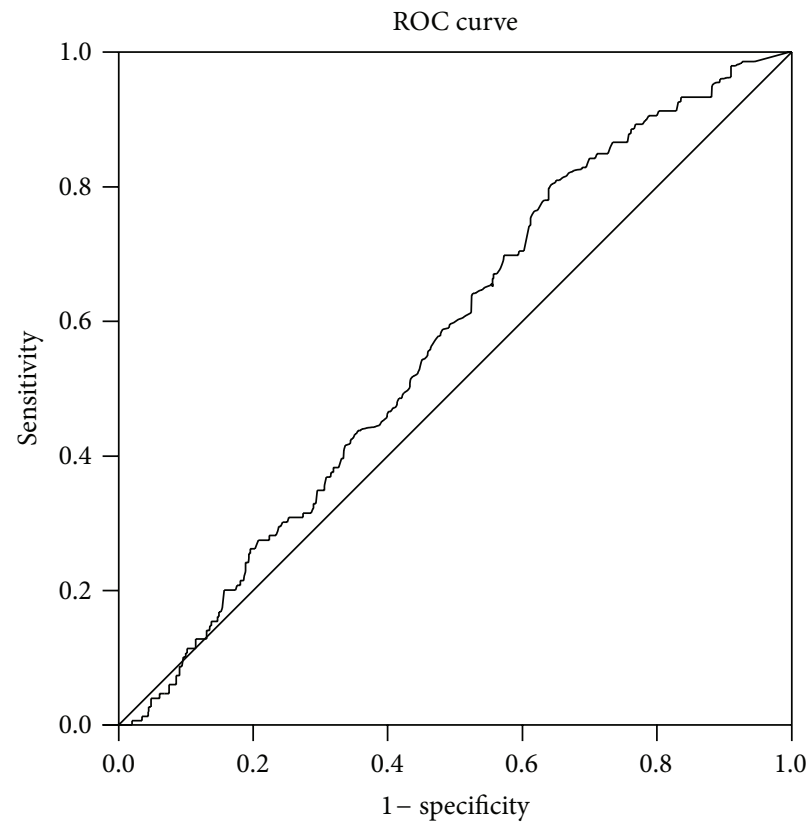

FIGURE 2: ROC curve for cancer prediction in a model for preoperative TSH testing.

concentrations $(p=0.001)$. All of the above analyses were repeated after all 149 patients with positive $\mathrm{TPOAb}$ or $\mathrm{TgAb}$ were removed, and the result did not change (Table 2).

ROC curve analysis was performed to determine the optimal TSH concentration for thyroid cancer prediction. It showed an area under the curve (AUC) of 0.58 (95\% CI 0.53$0.62, p=0.001)$, sensitivity of $74 \%$, and specificity of $57 \%$ at a cut-off of $1.59 \mathrm{mIU} / \mathrm{L}$ (Figure 2).

Furthermore, the TSH concentration was evaluated as a categorical variable within the following 5 ranges: $<0.27 \mathrm{mIU} /$ L (subclinical hyperthyroidism); $0.27-1.58 \mathrm{mIU} / \mathrm{L} ; 1.59$ (the cut-off value determined with the ROC curve) $-2.50 \mathrm{mIU} / \mathrm{L}$ (as more than $95 \%$ of normal individuals have TSH levels below $2.5 \mathrm{mIU} / \mathrm{L}$ ); $2.5-4.19 \mathrm{mIU} / \mathrm{L}$; and $\geq 4.2 \mathrm{mIU} / \mathrm{L}$ (subclinical hypothyroidism). The prevalence of DTC, according to the TSH concentration, indicated a clear TSH-related increase $(p<0.001$ and $p=0.014$, resp.). When the patients with positive autoantibodies were removed, the same pattern of escalating cancer incidence with increasing TSH persisted. In addition, among the divided TSH ranges, no significant difference was found between age groups ( $p=$ 0.339) (Figure 3).

When simultaneously analyzing sex, age, serum TSH concentration, TgAb, and TPOAb with binary logistic regression analysis, the results showed that the risk of DTC was 2.13fold higher if the TSH level was $1.59 \mathrm{mIU} / \mathrm{L}$ or greater relative to TSH levels less than $1.59 \mathrm{mIU} / \mathrm{L}$ (Table 3). A simultaneous likelihood ratio test of the effect of all these factors gives $\chi^{2}=$ 36.69 ( $p<0.001)$, indicating the combination of these factors for the prediction of malignancy to be very valuable.

FT3 and FT4 were also compared between patients with DTC and patients with BTND, but neither of them showed a significant difference, with or without the inclusion of the values out of the normal range (FT3, $p=0.77$; FT4, $p=0.91$ ).

3.3. TSH with the Progression of DTC. Figure 4 shows the association of serum TSH concentration and pathological characteristics in DTC. The prevalence of lymph node metastasis, extrathyroidal invasion, diffusion (spread in thyroid gland), and advanced stages (stages III and IV, according to 


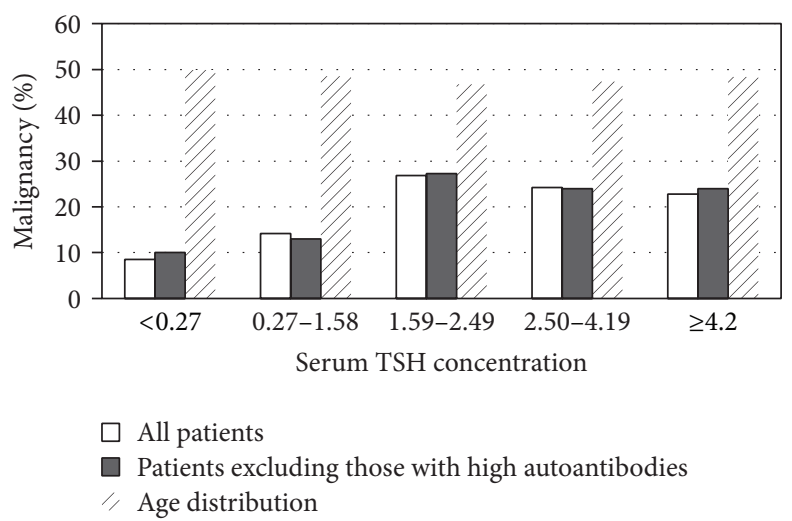

FIGURE 3: The distribution of patients based on TSH range and historical diagnosis.

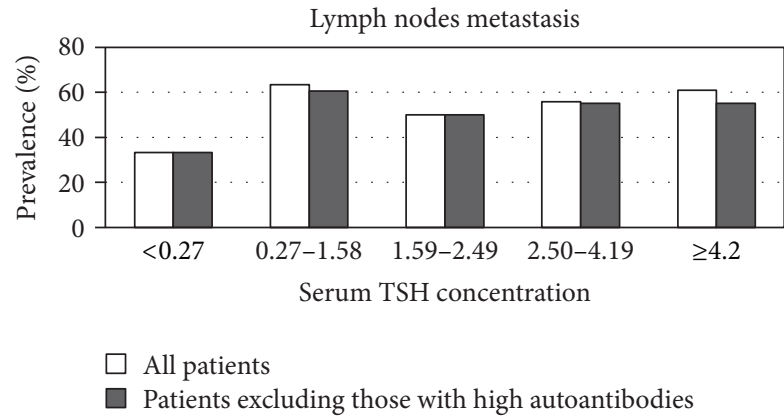

(a)

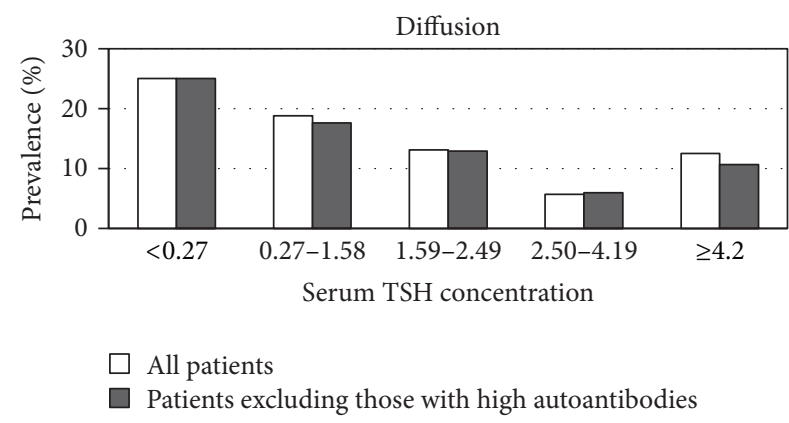

(c)

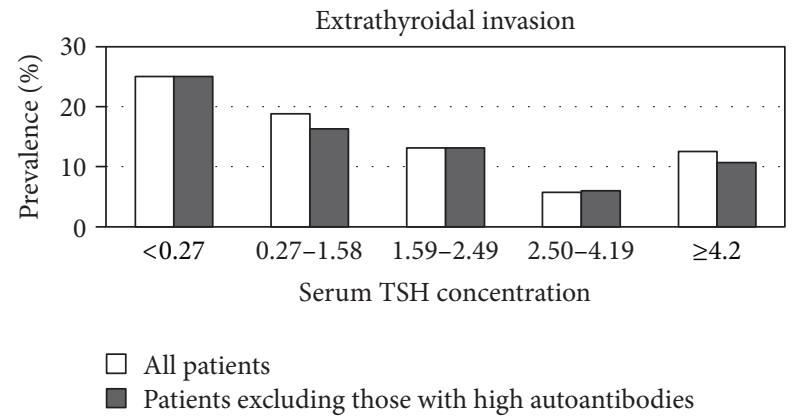

(b)

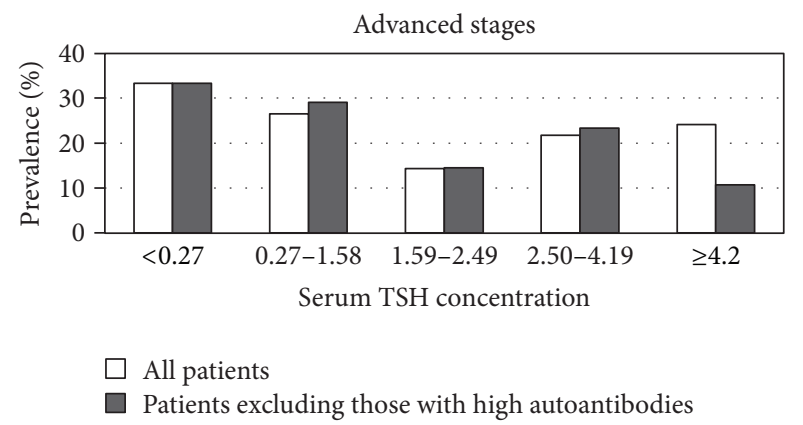

(d)

FIGURE 4: Association of serum TSH concentration and clinicopathological characteristics in differentiated thyroid cancer.

the TNM classification) were not related to TSH concentrations $(p>0.05)$. When the patients with positive TPOAb or $\mathrm{TgAb}$ were removed from the analysis, the results did not change.

3.4. Antibodies and Thyroid Carcinoma. After excluding patients with a history of exposure to levothyroxine or antithyroid drugs, 958 patients had available serum TPOAb results, and 937 had $\mathrm{TgAb}$ results.

The prevalence of malignancy was significantly higher in the TgAb-positive group than in the negative group $(p=$ $0.029, \mathrm{OR}=1.53$, and 95\% CI 1.04-2.24). There was a trend toward positive TPOAb and the prevalence of thyroid cancer, but it was not significant $(p=0.187)$, as shown in Table 4 . The binary logistic regression analysis (Table 3 ) indicated that the relationship between elevated $\operatorname{TgAb}$ and thyroid cancer did not persist after accounting for other variables $(p=0.11$, $\mathrm{OR}=1.53$, and $95 \% \mathrm{CI} 0.91-2.56$ ); the stepwise regression analysis showed that the association was lost when the effect of TSH was taken into account. In addition, TSH values were higher in patients with positive $\mathrm{TgAb}$ than in patients without $(6.32 \pm 1.03 \mathrm{mmol} / \mathrm{L}$ versus $2.43 \pm 0.15 \mathrm{mmol} / \mathrm{L}, p<0.001)$.

Both positive TPOAb and TgAb were closely correlated with pathologic HT $(p<0.001$ and $p<0.001$, resp.). Pathologic HT was detected in $10.92 \%$ (31/284) of patients with DTC and $11.0 \%$ (121/1105) of patients with BTND, which were not significantly different $(p=0.99)$.

3.5. Ultrasonography of Thyroid Carcinoma. Of the 1400 patients who underwent surgery for thyroid nodules between 
TABLE 2: Mean preoperative TSH.

\begin{tabular}{lccc}
\hline & $\begin{array}{c}\text { Number of } \\
\text { patients }\end{array}$ & $\begin{array}{c}\text { Mean TSH } \\
(\mathrm{mIU} / \mathrm{L})\end{array}$ & $p$ value \\
\hline All euthyroid patients $^{\mathrm{a}}$ & 164 & $2.10 \pm 0.07^{\mathrm{b}}$ & $0.004^{\mathrm{c}}$ \\
DTC & 111 & $2.18 \pm 0.09$ & $0.001^{\mathrm{d}}$ \\
TCLS & 53 & $1.94 \pm 0.12$ & \\
$\quad$ DTMCs & 630 & $1.86 \pm 0.04$ & \\
BTND & & & \\
Excluding patients with & & & \\
positive autoantibodies & 126 & $2.14 \pm 0.08$ & $<0.001^{\mathrm{c}}$ \\
DTC & 85 & $2.23 \pm 0.10$ & $<0.001^{\mathrm{d}}$ \\
TCLS & 41 & $1.96 \pm 0.14$ & \\
$\quad$ DTMCs & & & \\
BTND & 519 & $1.81 \pm 0.04$ & \\
Excluding patients with HT & & & \\
DTC & 151 & $2.11 \pm 0.07$ & $0.001^{\mathrm{c}}$ \\
$\quad$ TCLS & 104 & $2.19 \pm 0.09$ & $<0.001^{\mathrm{d}}$ \\
$\quad$ DTMCs & 47 & $1.93 \pm 0.13$ & \\
BTND & 586 & $1.80 \pm 0.04$ & \\
\hline
\end{tabular}

DTMCs: differentiated thyroid microcarcinomas; TCLS: thyroid cancers of larger size; HT: Hashimoto thyroiditis; BTND: benign thyroid nodular disease; and TSH: thyroid-stimulating hormone. ${ }^{a}$ Euthyroid based on TSH alone; ${ }^{b}$ described as mean $\pm \mathrm{SE}$; ${ }^{c}$ comparing group with DTC to group with BTND; ${ }^{\mathrm{d}}$ comparing among groups with TCLS, DTMCs, and benign diseases.

TABLE 3: Independent risk factors for the diagnosis of thyroid cancer defined by multivariable logistic regression analysis.

\begin{tabular}{lccc}
\hline Characteristics & $\begin{array}{c}\text { Adjusted } \\
\text { odds ratio }\end{array}$ & $\begin{array}{c}95 \% \\
\text { confidence } \\
\text { interval }\end{array}$ & $p$ value \\
\hline Male sex & 1.14 & $0.75-1.74$ & 0.531 \\
Age & 0.98 & $0.96-0.99$ & $<0.001$ \\
TSH $\geq 1.59 \mathrm{mIU} / \mathrm{L}$ & 2.13 & $1.48-3.07$ & $<0.001$ \\
TPOAb positivity & 0.92 & $0.54-1.57$ & 0.76 \\
TgAb positivity & 1.53 & $0.91-2.56$ & 0.11 \\
\hline
\end{tabular}

TSH: thyroid-stimulating hormone; TPOAb: thyroid peroxidase antibody; and TgAb: thyroglobulin antibody.

June 2008 and December 2010, 745 were excluded because images could not be retrieved for review. The remaining 655 patients comprised the study set for the evaluation of ultrasonographic features.

Of these 655 patients, $519(79.2 \%)$ patients had benign lesions and $136(20.8 \%)$ had malignant lesions. The prevalence of thyroid cancer did not differ among patients with a solitary thyroid nodule (51 of 236 patients, $21.6 \%$ ), two nodules (24 of 130 patients, 18.5\%), and multiple nodules (61 of 289 patients, $21.1 \%)(p=0.763)$.

A total of 374 patients had unilateral thyroid nodules, and the remaining 281 patients had bilateral thyroid nodules. The prevalence of thyroid cancer did not differ between the two groups (81 of 374 patients versus 55 of 281 patients, $p=0.515$ ).

It was reported that in patients with thyroid cancer in a gland with more than one nodule, $87 \%$ had cancer in the largest thyroid nodule [16], so we only recorded the largest nodule in each lobe to analyze the ultrasonographic features. There were 954 nodules in all: 447 nodules from left lobes, 474 from right lobes, and 33 from the isthmus, respectively. There was no significant difference in the thyroid cancer lobe distribution (left lobe, 78 of 447 patients; right lobe, 74 of 474 patients; and isthmus, 8 of 33 patients; $p=0.382$ ). The prevalence of thyroid cancer in each lobe did not differ between patients with a solitary nodule and patients with two or more nodules ( $p=0.538$ ), while those with multiple $(\geq 3)$ nodules had a lower likelihood of malignancy than those with less than three nodules $(p=0.008)$. Besides the number of nodules, the ultrasonographic characteristics that had a statistically significant association with thyroid cancer included nodular composition (solid component), echogenicity (hypoechoic), poorly defined margin, presence and type of blood flow (intranodular flow and peripheralintranodular flow), and punctate calcification (Table 5).

3.6. Ultrasonography of Thyroid Cancer of the Cervical Lymph Nodes. Using US, the lymph nodes of the neck were evaluated in 512 of 1400 patients. Among these, 440 patients were confirmed to have cervical lymphadenopathy, while the remaining 72 patients did not. The presence of cervical lymphadenopathy was related to a slightly higher prevalence of thyroid cancer (104 of 336 patients, $23.6 \%$ versus 12 of 72 patients, $16.7 \%)$, but the trend was not statistically significant $(p=0.19)$.

There was usually more than one cervical lymph node in each patient, and we only recorded the ultrasonographic characteristics of the lymph node that had the highest likelihood of metastasis in each patient to proceed to the next analysis. The study of ultrasonographic characteristics of cervical lymph nodes and histological diagnoses showed in Table 6.

\section{Discussion}

4.1. The Association between Serum TSH and Free Thyroid Hormone Concentrations in BTND and DTC Patients. Since Boelaert et al. [9] reported that TSH could be a risk factor for thyroid cancer in 2006, there have been many subsequent reports supporting their association [9-12, 17-20]. However, some studies made the final diagnosis depending on the FNAB results, which may be subject to ascertainment bias, as some patients may have been misdiagnosed $[9,10,12,17$, $18,21]$. In some studies, different thyroid malignancies were grouped together, including medullary, anaplastic cancers and thyroid lymphomas, which have never been reported to be TSH dependent $[9,10]$, and some studies of TSH values have been based on small patient populations $[10,19,20]$. This study largely overcame these limitations by using a large series of patients who underwent thyroid surgery with NTD, successfully further supporting the hypothesis that TSH is a risk predictor for DTC in Chinese patients. In patients who were euthyroid based on TSH levels alone, the mean serum TSH concentrations were significantly higher in patients with DTC compared to those with BTND, regardless of a history of taking levothyroxine. If DTC was subdivided into 
TABLE 4: Prevalence of thyroid cancer according to antibodies.

\begin{tabular}{|c|c|c|c|c|}
\hline Characteristics & Number of benign lesions (\%) & Number of malignant lesions (\%) & OR $(95 \% \mathrm{CI})$ & $p$ value \\
\hline TPOAb & & & $1.30(0.88-1.92)$ & 0.187 \\
\hline Positivity & $134(76.1 \%)$ & $42(23.9 \%)$ & & \\
\hline Negativity & $630(80.6 \%)$ & $152(19.4 \%)$ & & \\
\hline $\operatorname{TgAb}$ & & & $1.53(1.04-2.24)$ & 0.029 \\
\hline Positivity & $130(73.9 \%)$ & $46(26.1 \%)$ & & \\
\hline Negativity & $618(79.8 \%)$ & $143(20.2 \%)$ & & \\
\hline
\end{tabular}

OR: odds ratio; CI: confidence interval; TPOAb: thyroid peroxidase antibody; and TgAb: thyroglobulin antibody.

TABLE 5: Ultrasonographic characteristics of thyroid cancer.

\begin{tabular}{|c|c|c|c|c|}
\hline Characteristics & Number of benign nodules & Number of malignant nodules & $\%$ malignant & $p$ value \\
\hline Number of nodules & & & & 0.024 \\
\hline 1 & 404 & 93 & 18.7 & $0.538^{\mathrm{a}}$ \\
\hline 2 & 89 & 24 & 21.2 & $0.008^{\mathrm{b}}$ \\
\hline$\geq 3$ & 301 & 43 & 12.5 & \\
\hline Size, $m m($ mean \pm SE $)$ & $21.861 \pm 0.485$ & $23.295 \pm 1.198$ & & 0.235 \\
\hline Composition & & & & $<0.001$ \\
\hline Completely solid & 387 & 122 & 24.0 & \\
\hline Predominantly solid & 200 & 27 & 11.9 & \\
\hline Predominantly cystic & 131 & 6 & 4.4 & \\
\hline Completely cystic & 76 & 5 & 6.2 & \\
\hline Echogenicity & & & & $<0.001$ \\
\hline Hypoechoic & 273 & 100 & 26.8 & \\
\hline Hyperechoic & 111 & 21 & 15.9 & \\
\hline Isoechoic & 65 & 5 & 7.1 & \\
\hline Mixed echoic & 233 & 45 & 16.2 & \\
\hline Anechoic & 94 & 7 & 6.9 & \\
\hline Margin & & & & $<0.001$ \\
\hline Poorly defined & 136 & 68 & 33.3 & \\
\hline Well defined & 640 & 110 & 14.7 & \\
\hline Blood flow & & & & $<0.001$ \\
\hline Absent & 189 & 23 & 10.8 & \\
\hline Peripheral & 113 & 14 & 11.0 & \\
\hline Intranodular & 311 & 99 & 24.1 & \\
\hline Peripheral-intranodular & 164 & 41 & 20.0 & \\
\hline Calcification & & & & $<0.001$ \\
\hline None & 517 & 80 & 13.4 & \\
\hline Punctate & 200 & 82 & 29.1 & \\
\hline Coarse & 59 & 16 & 21.3 & \\
\hline Halo & & & & 0.109 \\
\hline None & 761 & 171 & 18.3 & \\
\hline Present & 15 & 7 & 31.8 & \\
\hline
\end{tabular}

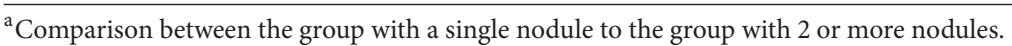

${ }^{\mathrm{b}}$ Comparison between the group with $<3$ nodules to the group with $\geq 3$ nodules.

DTMCs and TCLS, there was an incremental increase in TSH concentration in parallel with the tumor size, which implied that TSH could be not only a predictor of DTC, but also a parameter to determine the size of DTCs. This finding was similar to that in the study by Zafon et al., who found that the increase in TSH levels between the three groups (DTMCs, TCLS, and BTND) of patients was evident, but not statistically significant [22]. If the patients with positive autoantibodies were excluded, the result did not change, implying that the influence of TSH levels on tumorigenesis was not mediated through autoimmunity, corresponding with Fiore et al. [12]. The prevalence of DTC according to patients' TSH concentrations indicated a clear TSH-related increase when we evaluated the TSH concentration as a 
TABLE 6: Ultrasonographic characteristics of cervical lymph nodes and histological diagnoses.

\begin{tabular}{|c|c|c|c|c|c|c|c|c|}
\hline \multirow{2}{*}{ Ultrasonographic characteristics } & \multicolumn{4}{|c|}{ Histological diagnosis of thyroid nodules } & \multicolumn{4}{|c|}{ Lymph nodes metastasis } \\
\hline & Benign & Malignant & OR (95\% CI) & $p$ & Present & Absent & OR $(95 \% \mathrm{CI})$ & $p$ \\
\hline Longest diameter & & & $2.55(1.47-4.42)$ & 0.001 & & & $1.86(0.54-6.44)$ & NS \\
\hline$\geq 15 \mathrm{~mm}$ & 191 & 77 & & & 40 & 27 & & \\
\hline$<15 \mathrm{~mm}$ & 120 & 19 & & & 11 & 4 & & \\
\hline Shortest diameter & & & $2.08(1.28-3.37)$ & 0.003 & & & $0.84(0.31-2.27)$ & NS \\
\hline$\geq 5 \mathrm{~mm}$ & 160 & 66 & & & 38 & 22 & & \\
\hline$<5 \mathrm{~mm}$ & 151 & 30 & & & 13 & 9 & & \\
\hline $\mathrm{L} / \mathrm{S} \mathrm{ratio}^{\mathrm{a}}$ & & & $4.11(2.14-7.87)$ & $<0.001$ & & & $3.38(1.02-11.21)$ & 0.04 \\
\hline$>2$ & 290 & 74 & & & 17 & 4 & & \\
\hline$<2$ & 21 & 22 & & & 34 & 27 & & \\
\hline Margins & & & $3.34(0.47-24.04)$ & NS & & & $1.66(0.1-27.41)$ & NS \\
\hline Blurred & 2 & 2 & & & 1 & 1 & & \\
\hline Defined & 331 & 99 & & & 53 & 32 & & \\
\hline Fusion & & & $24.80(3.01-204.08)$ & $<0.001$ & & & $1.70(1.42-2.05)$ & 0.031 \\
\hline Present & 1 & 7 & & & 7 & 0 & & \\
\hline Absent & 333 & 94 & & & 47 & 33 & & \\
\hline Vascularity & & & $2.03(1.26-3.26)$ & 0.003 & & & $0.59(0.24-1.48)$ & NS \\
\hline Present & 176 & 70 & & & 39 & 20 & & \\
\hline Absent & 158 & 31 & & & 15 & 13 & & \\
\hline Calcification & & & $48.39(6.25-374.84)$ & $<0.001$ & & & $0.28(0.06-1.39)$ & NS \\
\hline Present & 0 & 12 & & & 10 & 2 & & \\
\hline Absent & 334 & 89 & & & 44 & 31 & & \\
\hline
\end{tabular}

${ }^{a} \mathrm{~L} / \mathrm{S}$ ratio: large axis to small axis ratio.

categorical variable within five ranges, and the incidence of DTC increased significantly when the TSH concentration was higher than $1.59 \mathrm{mIU} / \mathrm{L}$. Our statistics also showed that there was no significant difference in the age distribution among the divided five ranges of TSH concentrations, making it clear that the significant relationship was not based on age. Levothyroxine-treated patients with respect to untreated patients showed a markedly lower prevalence of DTC, although the trend did not reach statistical significance. TSH showed a sensitivity of $74 \%$ and specificity of $57 \%$ at a cutoff of $1.59 \mathrm{mIU} / \mathrm{L}$ determined with ROC curve analysis, which showed an AUC of 0.58 (95\% CI 0.53-0.62, $p=0.001)$. When simultaneously analyzing sex, age, TgAb, TPOAb, and serum TSH concentration by binary logistic regression analysis, a 2.13-fold risk of DTC was shown when the TSH concentration was $1.59 \mathrm{mIU} / \mathrm{L}$ or greater, relative to TSH concentrations less than $1.59 \mathrm{mIU} / \mathrm{L}$.

Our study failed to show a significant effect of serum TSH concentration on the prognosis of DTC patients. In patients with DTC, the prevalence of lymph node metastases, extrathyroidal invasion, diffusion, and advanced stages (stages III and IV) were not related to TSH concentrations. Some previous research has shown similar results to ours [23]. However, other researchers have shown that higher serum TSH levels were associated with prognostic markers of DTC, including cancer stage, tumor size, lymph node status, extrathyroidal extension, and distant metastases [11, 12, 24]. Clearly, further studies are required as there is still some debate.
In our study, both FT3 and FT4 were not associated with DTC; this result was consistent with some previous studies $[12,14]$. Although a prior study has shown that TT3 was associated with DTC [19], the author did not use a separate test for FT3. As the test of TT3 not only reflects the level of FT3 but is also influenced by the level of thyroid binding globulin, this result cannot further demonstrate the association between triiodothyronine and the incidence of thyroid cancer.

4.2. Thyroid Antibodies and Thyroid Cancer. In our study, a significantly higher prevalence of cancer was found in patients with serum positive TgAb compared to those with negative $\mathrm{TgAb}$, but not TPOAb, a more specific serum marker of full-blown HT [25]. Unlike some other researches that also showed that $\mathrm{TgAb}$ was a predictor for thyroid cancer $[23,26]$, in our study, the association between $\mathrm{TgAb}$ and thyroid cancer no longer existed when the effect of TSH was taken into account. Furthermore, we also showed that the TSH concentration was significantly higher in patients with positive TgAb than in patients with negative TgAb. A previous study showed that the presence of thyroid autoantibodies was associated with a significant increase in TSH, but it did not mention TgAb separately [12]. Based on the results above, TSH may explain the association between positive $\mathrm{TgAb}$ and thyroid malignancy; further studies are necessary to clarify this point. The association between TPOAb and thyroid cancer was not found in our study, consistent with some prior studies $[9,27]$. It is noteworthy that a large study 
of palpable thyroid nodules found that TPOAb was associated with an increased risk of malignancy, but this association was lost when the effect of TSH was taken into account [9].

Many studies have discovered a strong association between HT (assessed by the presence of lymphocytic thyroiditis and/or thyroid autoantibodies) and thyroid cancer $[28,29]$. It has been reported that there was more lymphocytic thyroiditis in malignant nodules than in benign ones [30]. A recent study also showed that the chronic inflammation that is associated with lymphocytic thyroiditis has the potential to activate cytokines and growth factors and ultimately promote tumorigenesis [31]. Furthermore, HT and PTC share genetic and biomolecular characteristics such as RET/PTC rearrangements [32] and the expression of P63 [33] and Akt proteins [34] that are thought to be involved in neoplastic transformation. In addition, the chronic TSH stimulation secondary to HT could be another factor that might promote tumorigenesis. Even so, some other studies opposed the association of HT with thyroid cancer. Particularly, two large prospective studies with a follow-up over 10 years failed to reveal a higher incidence of thyroid cancer in goiters with HT compared with goiters without HT [35, 36]. Our study also showed that HT was not predictive of malignancy through the following two results: (1) TPOAb, a more specific serum marker of full-blown HT, did not show a significant association with thyroid cancer; (2) there was no significant difference in the frequency of pathologic HT in DTC and BTND specimens. Therefore, this result further suggests that the expression of a coexistent HT cannot account for the significantly increased prevalence of cancer in patients with positive $\mathrm{TgAb}$.

4.3. US of Thyroid Cancer. As the most common imaging examination for thyroid nodules, US has the advantage of being widely available, well tolerated, affordable, and lowrisk. Even though ultrasound alone cannot reliably distinguish malignant and benign lesions, some features have been consistently associated with malignancy according to some researches [37-41]. Our study is the first to investigate the ultrasonographic features of lobe units. There was no significant difference in the prevalence of thyroid malignancy among left lobe, right lobe, and isthmus. By recording the largest nodule in each lobe, our results showed that nodule size was not a predictor of thyroid malignancy. It was reported that a large tumor size indicated an increased risk of malignancy [42-44], while some other researches showed the opposite effect $[45,46]$. In addition, a prospective study has shown that increasing nodule size is not predictive of thyroid malignancy [47].

Our results showed that multiple $(\geq 3)$ nodules had a lower likelihood of malignancy than in cases with less than three nodules, but we failed to show that a single nodule carries an increased risk of thyroid cancer. Previous studies reporting single nodules identified with US [27, 48] showed similar results to ours, while other studies reporting single nodules identified with a physical examination [9] showed results contrary to ours; these differences may be due to the increasing sensitivity of thyroid US, as it was reported that approximately $23 \%$ of palpable solitary nodules are actually dominant nodules within a multinodular goiter [49].

Our results also showed that the presence of punctate calcification, solid composition (the more solid a nodule was, the more likely it was to be malignant), hypoechoic regions, and poorly defined margins and the presence and type of blood flow (intranodular flow and peripheral-intranodular flow) were all significant predictors for thyroid cancer, consistent with prior studies [37-41]. The presence of a halo around the nodule was unrelated to the risk of malignancy, consistent with some previous reports $[16,50]$, but inconsistent with others $[51,52]$.

\subsection{US of Cervical Lymph Nodes with Thyroid Cancer.} Cervical lymph nodes are involved in a number of disease conditions. For NTD patients, the most common causes of cervical lymphadenopathy are metastasis and reactive lymph nodes. US is increasingly being recognized as a noninvasive tool for the evaluation of cervical lymph nodes. It is known that, in $20 \%-50 \%$ of patients with DTC, the cervical lymph nodes can be involved $[53,54]$. Our results showed that the presence of cervical lymphadenopathy in NTD patients was not significantly suggestive of thyroid malignancy $(p=0.19)$, but some ultrasonographic characteristics of lymph nodes were of great significance. A L/S ratio $<2$, which indicated a more spherical shape, was not only strongly suggestive of malignancy in NTD patients, but it was also suggestive of metastasis in DTC patients, in agreement with prior studies $[55,56]$. In addition, the longest diameter $\geq 15 \mathrm{~mm}$, the shortest diameter $\geq 5 \mathrm{~mm}$, and the presence of fusion, vascularity, and calcification all indicated the presence of malignancy in NTD patients, but they were not able to differentiate cases with and without metastatic lymph nodes. However, blurred margins were not a distinguishing feature of the lymph nodes in patients with a thyroid malignancy, contrary to what was observed in prior studies [56].

4.5. Age and Sex in Thyroid Cancer. Multiple populationbased studies have shown age to be an independent risk factor for thyroid cancer [11, 21]; this was confirmed in our study, which revealed that the patients with malignancies were significantly younger compared to those without malignancies. Furthermore, a statistically significant higher rate of malignancy in patients who were under 40 and over 70 years of age was observed in our study. In Western countries, age under 20 and over 70 years was considered a risk factor for thyroid cancer; patients over the age of 20 had a relatively low risk of thyroid cancer compared to patients under the age of 20 years [9]. On the contrary, we found that patients who were 20-40 years of age had a higher risk of thyroid cancer compared to patients younger than 20 years of age. More evidence is needed to clarify whether this discrepancy is caused by population-based differences.

Whether the male sex is a risk factor for thyroid cancer is still controversial. Although a number of research results suggested that men have a higher incidence of thyroid cancer than women do [9-11, 21], some other researches have found that sex did not have a direct impact on the incidence of 
thyroid cancer $[57,58]$; Alexander et al. conducted a followup examination 1 month to 5 years after diagnosis for 1009 patients who were treated for benign thyroid nodules, and their result showed that sex did not predict thyroid nodule growth [59]. Our results showed that there was no significant difference in the prevalence of thyroid cancer between men and women.

\section{Additional Points}

We acknowledge the following limitations of this study: (1) retrospective studies of pathology may be subject to ascertainment bias; (2) in our study, only the patients who underwent surgery for NTD were included, creating a selection bias, as surgery was only performed for the NTD patients whose nodules were considered to have the possibility of malignancy; and (3) we only recorded the largest thyroid nodules in each thyroid lobe and the lymph node that had the greatest likelihood of metastasis based on US, so we have inevitably missed some important information.

\section{Competing Interests}

The authors declare that they have no competing interests.

\section{References}

[1] E. L. Mazzaferri, "Thyroid cancer in thyroid nodules: finding a needle in the haystack," The American Journal of Medicine, vol. 93, no. 4, pp. 359-362, 1992.

[2] G. H. Tan and H. Gharib, "Thyroid incidentalomas: management approaches to nonpalpable nodules discovered incidentally on thyroid imaging," Annals of Internal Medicine, vol. 126, no. 3, pp. 226-231, 1997.

[3] S. Noguchi, H. Yamashita, S. Uchino, and S. Watanabe, "Papillary microcarcinoma," World Journal of Surgery, vol. 32, no. 5, pp. 747-753, 2008.

[4] T. Kimura, A. Van Keymeulen, J. Golstein, A. Fusco, J. E. Dumont, and P. P. Roger, "Regulation of thyroid cell proliferation by TSH and other factors: a critical evaluation of in vitro models," Endocrine Reviews, vol. 22, no. 5, pp. 631-656, 2001.

[5] A. Samadi, P. Loo, R. Mukerji et al., "A novel HSP90 modulator with selective activity against thyroid cancers in vitro," Surgery, vol. 146, no. 6, pp. 1196-1207, 2009.

[6] M. Ariga, T. Nedachi, M. Akahori et al., "Signalling pathways of insulin-like growth factor-I that are augmented by cAMP in FRTL-5 cells," Biochemical Journal, vol. 348, no. 2, pp. 409-416, 2000.

[7] D. S. Cooper, G. M. Doherty, B. R. Haugen et al., "Management guidelines for patients with thyroid nodules and differentiated thyroid cancer," Thyroid, vol. 16, no. 2, pp. 109-142, 2006.

[8] P. Perros, K. Boelaert, S. Colley et al., "Guidelines for the management of thyroid cancer," Clinical Endocrinology, vol. 81, supplement 1, pp. 1-122, 2014.

[9] K. Boelaert, J. Horacek, R. L. Holder, J. C. Watkinson, M. C. Sheppard, and J. A. Franklyn, "Serum thyrotropin concentration as a novel predictor of malignancy in thyroid nodules investigated by fine-needle aspiration," Journal of Clinical Endocrinology and Metabolism, vol. 91, no. 11, pp. 4295-4301, 2006.
[10] S. A. Polyzos, M. Kita, Z. Efstathiadou et al., "Serum thyrotropin concentration as a biochemical predictor of thyroid malignancy in patients presenting with thyroid nodules," Journal of Cancer Research and Clinical Oncology, vol. 134, no. 9, pp. 953-960, 2008.

[11] M. R. Haymart, D. J. Repplinger, G. E. Leverson et al., "Higher serum thyroid stimulating hormone level in thyroid nodule patients is associated with greater risks of differentiated thyroid cancer and advanced tumor stage," Journal of Clinical Endocrinology and Metabolism, vol. 93, no. 3, pp. 809-814, 2008.

[12] E. Fiore, T. Rago, M. A. Provenzale et al., "Lower levels of TSH are associated with a lower risk of papillary thyroid cancer in patients with thyroid nodular disease: thyroid autonomy may play a protective role," Endocrine-Related Cancer, vol. 16, no. 4, pp. 1251-1260, 2009.

[13] E. Fiore and P. Vitti, "Serum TSH and risk of papillary thyroid cancer in nodular thyroid disease," Journal of Clinical Endocrinology and Metabolism, vol. 97, no. 4, pp. 1134-1145, 2012.

[14] K. W. Kim, Y. J. Park, E. H. Kim et al., "Elevated risk of papillary thyroid cancer in Korean patients with Hashimoto's thyroiditis," Head and Neck, vol. 33, no. 5, pp. 691-695, 2011.

[15] M. R. Castro, R. P. Espiritu, R. S. Bahn et al., "Predictors of malignancy in patients with cytologically suspicious thyroid nodules," Thyroid, vol. 21, no. 11, pp. 1191-1198, 2011.

[16] M. C. Frates, C. B. Benson, P. M. Doubilet et al., "Prevalence and distribution of carcinoma in patients with solitary and multiple thyroid nodules on sonography," Journal of Clinical Endocrinology and Metabolism, vol. 91, no. 9, pp. 3411-3417, 2006.

[17] E. Fiore, T. Rago, M. A. Provenzale et al., "L-thyroxinetreated patients with nodular goiter have lower serum TSH and lower frequency of papillary thyroid cancer: results of a crosssectional study on 27914 patients," Endocrine-Related Cancer, vol. 17, no. 1, pp. 231-239, 2010.

[18] E. Fiore, T. Rago, F. Latrofa et al., "Hashimoto's thyroiditis is associated with papillary thyroid carcinoma: role of TSH and of treatment with L-thyroxine," Endocrine-Related Cancer, vol. 18 , no. 4, pp. 429-437, 2011.

[19] J. Jonklaas, H. Nsouli-Maktabi, and S. J. Soldin, "Endogenous thyrotropin and triiodothyronine concentrations in individuals with thyroid cancer," Thyroid, vol. 18, no. 9, pp. 943-952, 2008.

[20] K. Gul, D. Ozdemir, A. Dirikoc et al., "Are endogenously lower serum thyroid hormones new predictors for thyroid malignancy in addition to higher serum thyrotropin?" Endocrine, vol. 37, no. 2, pp. 253-260, 2010.

[21] T. Rago, E. Fiore, M. Scutari et al., "Male sex, single nodularity, and young age are associated with the risk of finding a papillary thyroid cancer on fine-needle aspiration cytology in a large series of patients with nodular thyroid disease," European Journal of Endocrinology, vol. 162, no. 4, pp. 763-770, 2010.

[22] C. Zafon, G. Obiols, J. A. Baena, J. Castellví, B. Dalama, and J. Mesa, "Preoperative thyrotropin serum concentrations gradually increase from benign thyroid nodules to papillary thyroid microcarcinomas then to papillary thyroid cancers of larger size," Journal of Thyroid Research, vol. 2012, Article ID 530721, 4 pages, 2012.

[23] E. S. Kim, D. J. Lim, K. H. Baek et al., “Thyroglobulin antibody is associated with increased cancer risk in thyroid nodules," Thyroid, vol. 20, no. 8, pp. 885-891, 2010. 
[24] S. S. Kim, B. J. Lee, J. C. Lee et al., "Preoperative serum thyroid stimulating hormone levels in well-differentiated thyroid carcinoma is a predictive factor for lateral lymph node metastasis as well as extrathyroidal extension in Korean patients: a singlecenter experience," Endocrine, vol. 39, no. 3, pp. 259-265, 2011.

[25] S. Mariotti, P. Caturegli, P. Piccolo, G. Barbesino, and A. Pinchera, "Antithyroid peroxidase autoantibodies in thyroid disease," Journal of Clinical Endocrinology and Metabolism, vol. 71, no. 3, pp. 661-669, 1990.

[26] T. Rago, G. Di Coscio, C. Ugolini et al., "Clinical features of thyroid autoimmunity are associated with thyroiditis on histology and are not predictive of malignancy in 570 patients with indeterminate nodules on cytology who had a thyroidectomy," Clinical Endocrinology, vol. 67, no. 3, pp. 363-369, 2007.

[27] G. Azizi and C. D. Malchoff, "Autoimmune thyroid disease: a risk factor for thyroid cancer," Endocrine Practice, vol. 17, no. 2, pp. 201-209, 2011.

[28] L. Zhang, H. Li, Q.-H. Ji et al., “The clinical features of papillary thyroid cancer in Hashimoto's thyroiditis patients from an area with a high prevalence of Hashimoto's disease," BMC Cancer, vol. 12, article 610, 2012.

[29] A. Konturek, M. Barczyński, W. Wierzchowski, M. Stopa, and W. Nowak, "Coexistence of papillary thyroid cancer with Hashimoto thyroiditis," Langenbeck's Archives of Surgery, vol. 398, no. 3, pp. 389-394, 2013.

[30] I. Okayasu, M. Fujiwara, Y. Hara, Y. Tanaka, and N. R. Rose, "Association of chronic lymphocytic thyroiditis and thyroid papillary carcinoma: a study of surgical cases among Japanese, and white and African Americans," Cancer, vol. 76, no. 11, pp. 2312-2318, 1995.

[31] V. Guarino, M. D. Castellone, E. Avilla, and R. M. Melillo, "Thyroid cancer and inflammation," Molecular and Cellular Endocrinology, vol. 321, no. 1, pp. 94-102, 2010.

[32] A. Wirtschafter, R. Schmidt, D. Rosen et al., "Expression of the RET/PTC fusion gene as a marker for papillary carcinoma in Hashimoto's thyroiditis," Laryngoscope, vol. 107, no. 1, pp. 95100, 1997.

[33] P. Unger, M. Ewart, B. Y. Wang, L. Gan, D. S. Kohtz, and D. E. Burstein, "Expression of p63 in papillary thyroid carcinoma and in hashimoto's thyroiditis: a pathobiologic link?" Human Pathology, vol. 34, no. 8, pp. 764-769, 2003.

[34] S. D. Larson, L. N. Jackson, T. S. Riall et al., "Increased incidence of well-differentiated thyroid cancer associated with Hashimoto thyroiditis and the role of the PI3k/Akt pathway," Journal of the American College of Surgeons, vol. 204, no. 5, pp. 764-773, 2007.

[35] G. Crile Jr., "Struma lymphomatosa and carcinoma of the thyroid," Surgery Gynecology and Obstetrics, vol. 147, no. 3, pp. 350-352, 1978.

[36] L. E. Holm, H. Blomgren, and T. Lowhagen, "Cancer risks in patients with chronic lymphocytic thyroiditis," The New England Journal of Medicine, vol. 312, no. 10, pp. 601-604, 1985.

[37] E.-K. Kim, S. P. Cheong, Y. C. Woung et al., "New sonographic criteria for recommending fine-needle aspiration biopsy of nonpalpable solid nodules of the thyroid," American Journal of Roentgenology, vol. 178, no. 3, pp. 687-691, 2002.

[38] J. K. Hoang, K. L. Wai, M. Lee, D. Johnson, and S. Farrell, "US features of thyroid malignancy: pearls and pitfalls," Radiographics, vol. 27, no. 3, pp. 847-860, 2007.

[39] W.-J. Moon, L. J. So, H. L. Jeong et al., "Benign and malignant thyroid nodules: US differentiation-multicenter retrospective study," Radiology, vol. 247, no. 3, pp. 762-770, 2008.
[40] D. S. Cooper, G. M. Doherty, B. R. Haugen et al., "Revised American Thyroid Association management guidelines for patients with thyroid nodules and differentiated thyroid cancer," Thyroid, vol. 19, no. 11, pp. 1167-1214, 2009.

[41] S. S. Ahn, E.-K. Kim, D. R. Kang, S.-K. Lim, J. Y. Kwak, and M. J. Kim, "Biopsy of thyroid nodules: comparison of three sets of guidelines," American Journal of Roentgenology, vol. 194, no. 1, pp. 31-37, 2010.

[42] R. S. Sippel, D. M. Elaraj, E. Khanafshar et al., "Tumor size predicts malignant potential in Hürthle cell neoplasms of the thyroid," World Journal of Surgery, vol. 32, no. 5, pp. 702-707, 2008.

[43] K. Raparia, S. K. Min, D. R. Mody, R. Anton, and M. Amrikachi, "Clinical outcomes for suspicious category in thyroid fineneedle aspiration biopsy patient's sex and nodule size are possible predictors of malignancy," Archives of Pathology and Laboratory Medicine, vol. 133, no. 5, pp. 787-790, 2009.

[44] N. Besic, M. Sesek, B. Peric, J. Zgainar, and M. Hocevar, "Predictive factors of carcinoma in 327 patients with follicular neoplasm of the thyroid," Medical Science Monitor, vol. 14, no. 9, pp. R459-R467, 2008.

[45] M. Kihara, M. Hirokawa, H. Masuoka et al., "Evaluation of cytologically benign solitary thyroid nodules by ultrasonography: a retrospective analysis of 1877 cases," Auris Nasus Larynx, vol. 40, no. 3, pp. 308-311, 2013.

[46] G. Anil, A. Hegde, and F. H. V. Chong, "Thyroid nodules: risk stratification for malignancy with ultrasound and guided biopsy," Cancer Imaging, vol. 11, no. 1, pp. 209-223, 2011.

[47] C. R. McHenry, E. S. Huh, and R. N. Machekano, "Is nodule size an independent predictor of thyroid malignancy?" Surgery, vol. 144, no. 6, pp. 1062-1069, 2008.

[48] E. Papini, R. Guglielmi, A. Bianchini et al., "Risk of malignancy in nonpalpable thyroid nodules: predictive value of ultrasound and color-doppler features," Journal of Clinical Endocrinology and Metabolism, vol. 87, no. 5, pp. 1941-1946, 2002.

[49] R. M. Walsh, J. C. Watkinson, and J. Franklyn, "The management of the solitary thyroid nodule: a review," Clinical Otolaryngology and Allied Sciences, vol. 24, no. 5, pp. 388-397, 1999.

[50] W.-W. Zhan, P. Zhou, J.-Q. Zhou, S.-Y. Xu, and K.-M. Chen, "Differences in sonographic features of papillary thyroid carcinoma between neck lymph node metastatic and nonmetastatic groups," Journal of Ultrasound in Medicine, vol. 31, no. 6, pp. 915920, 2012.

[51] A. S. Kabaker, M. E. Tublin, Y. E. Nikiforov et al., "Suspicious ultrasound characteristics predict BRAFV600E-positive papillary thyroid carcinoma," Thyroid, vol. 22, no. 6, pp. 585-589, 2012.

[52] M.-S. Ko, K. S. Jeong, Y. K. Shong, G. Y. Gong, J. H. Baek, and J. H. Lee, "Collapsing benign cystic nodules of the thyroid gland: sonographic differentiation from papillary thyroid carcinoma," American Journal of Neuroradiology, vol. 33, no. 1, pp. 124-127, 2012.

[53] G. F. W. Scheumann, O. Gimm, G. Wegener, H. Hundeshagen, and H. Dralle, "Prognostic significance and surgical management of locoregional lymph node metastases in papillary thyroid cancer," World Journal of Surgery, vol. 18, no. 4, pp. 559567, 1994.

[54] S.-M. Chow, S. C. K. Law, J. K. C. Chan, S.-K. Au, S. Yau, and W.H. Lau, "Papillary microcarcinoma of the thyroid-prognostic significance of lymph node metastasis and multifocality," Cancer, vol. 98, no. 1, pp. 31-40, 2003. 
[55] H. J. Steinkamp, M. Cornehl, N. Hosten, W. Pegios, T. Vogl, and R. Felix, "Cervical lymphadenopathy: ratio of long- to shortaxis diameter as a predictor of malignancy," British Journal of Radiology, vol. 68, no. 807, pp. 266-270, 1995.

[56] R. Khanna, A. D. Sharma, S. Khanna, M. Kumar, and R. C. Shukla, "Usefulness of ultrasonography for the evaluation of cervical lymphadenopathy," World Journal of Surgical Oncology, vol. 9, article 29, 2011.

[57] C. R. McHenry, P. G. Walfish, and I. B. Rosen, "Non-diagnostic fine needle aspiration biopsy: a dilemma in management of nodular thyroid disease," American Surgeon, vol. 59, no. 7, pp. 415-419, 1993.

[58] W. Raber, K. Kaserer, B. Niederle, and H. Vierhapper, "Risk factors for malignancy of thyroid nodules initially identified as follicular neoplasia by fine-needle aspiration: results of a prospective study of one hundred twenty patients," Thyroid, vol. 10, no. 8, pp. 709-712, 2000.

[59] E. K. Alexander, S. Hurwitz, J. P. Heering et al., "Natural history of benign solid and cystic thyroid nodules," Annals of Internal Medicine, vol. 138, no. 4, pp. 315-318, 2003. 


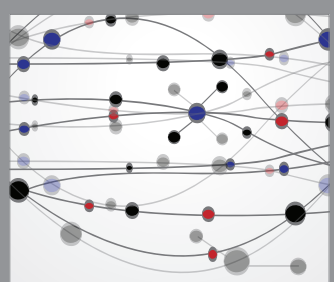

The Scientific World Journal
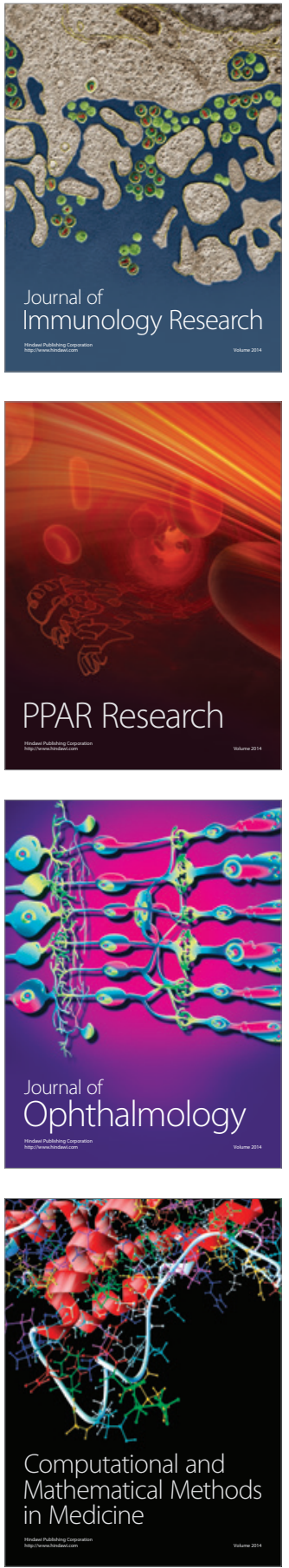

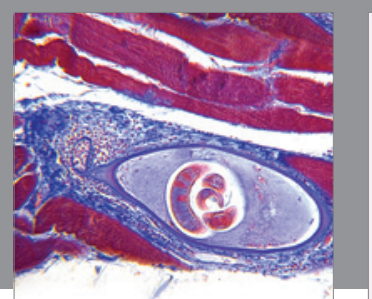

Gastroenterology Research and Practice

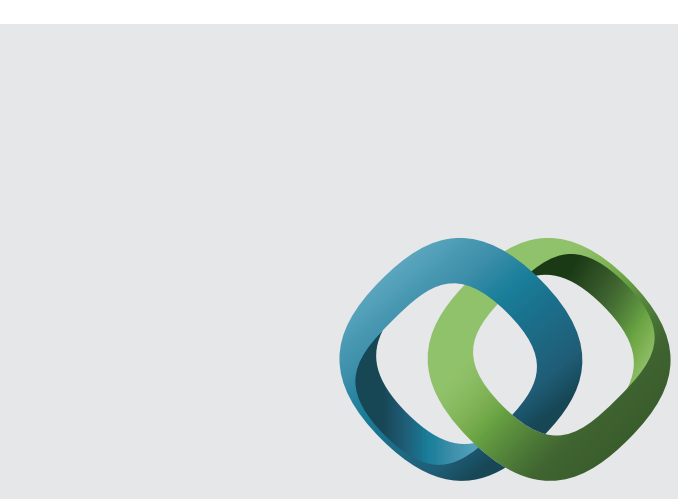

\section{Hindawi}

Submit your manuscripts at

http://www.hindawi.com
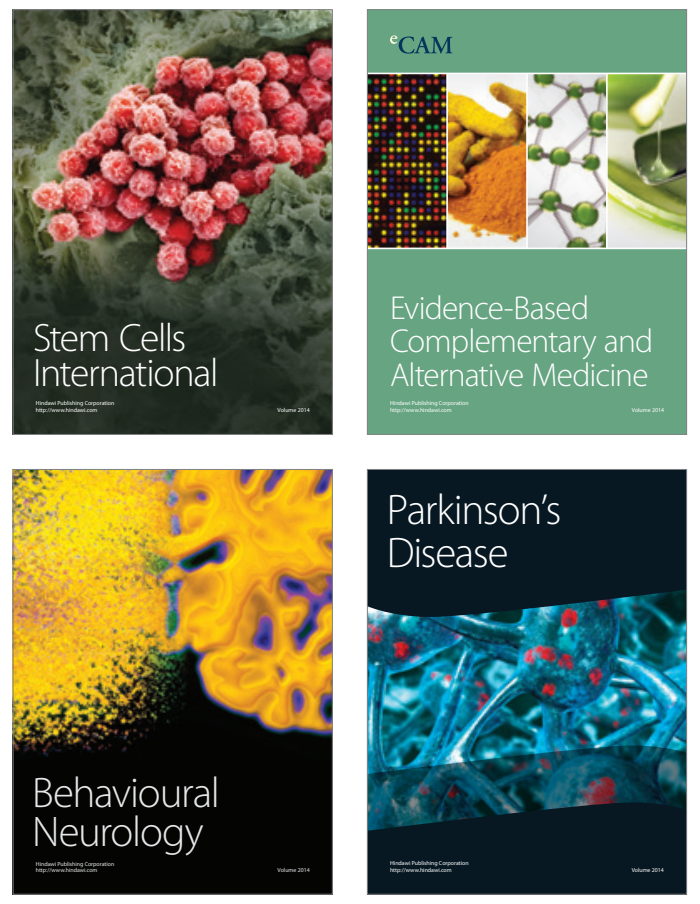
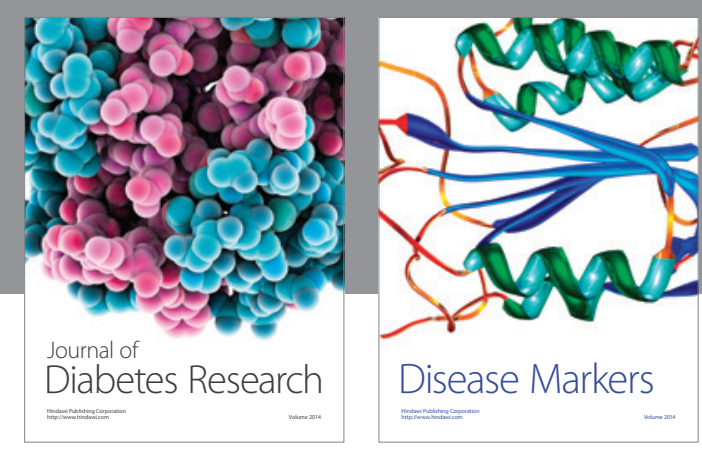

Disease Markers
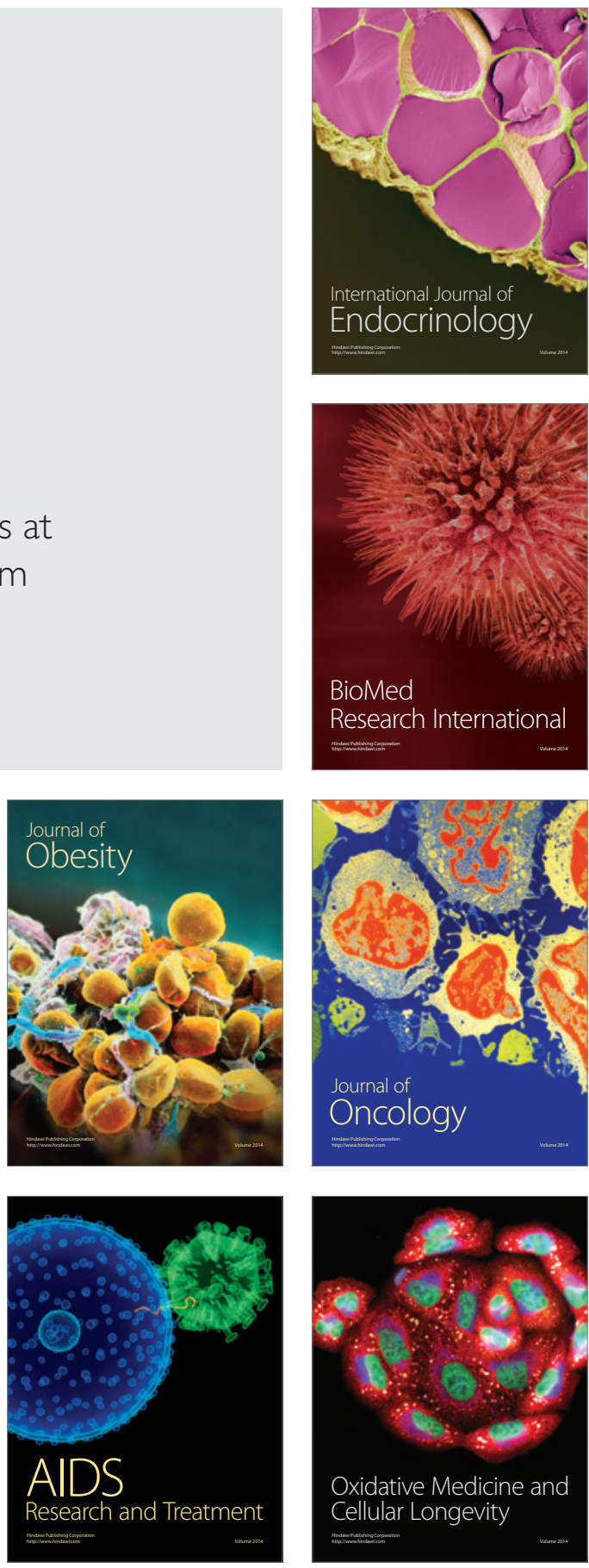